\title{
Magnetic Anisotropy Variations and Non-Equilibrium Tunneling in a Cobalt Nanoparticle
}

\author{
M. M. Deshmukh ${ }^{1}$, S. Guéron ${ }^{2}$, E. Bonet ${ }^{1}$, A. N. Pasupathy ${ }^{1}$, S. Kleff ${ }^{3,4}$, J. von Delft ${ }^{3,4}$, and D. C. Ralph ${ }^{1}$ \\ ${ }^{1}$ Laboratory of Atomic and Solid State Physics, Cornell University, Ithaca, NY, 14853 \\ ${ }^{2}$ Laboratoire de Physique des Solides, Université Paris Sud, 91405 Orsay, France \\ ${ }^{3}$ Institut für Theoretische Festkörperphysik, Universität Karlsruhe, 76128 Karlsruhe, Germany \\ ${ }^{4}$ Ludwig-Maximilians-Universität, Theresienstr. 37, 80333 München, Germany
}

(November 2, 2018)

\begin{abstract}
We present detailed measurements of the discrete electron-tunneling level spectrum within nanometer-scale cobalt particles as a function of magnetic field and gate voltage, in this way probing individual quantum many-body eigenstates inside ferromagnetic samples. Variations among the observed levels indicate that different quantum states within one particle are subject to different magnetic anisotropy energies. Gate-voltage studies demonstrate that the low-energy tunneling spectrum is affected dramatically by the presence of non-equilibrium spin excitations.
\end{abstract}

PACS numbers: 73.22.-f, 73.23.Hk, 75.75.+a

The electronic states within ferromagnetic devices are understood surprisingly poorly. For example, several types of experiments have been done to characterize spin polarization near the Fermi level [1 15], but different techniques give different results. Part of the difficulty is that these experiments average over large numbers of states, and different types of experiments effectively take differently weighted averages. Recently, we have developed a tunneling technique that can resolve the individual states within nm-scale samples of ferromagnets [6]. This method has helped motivate new descriptions of ferromagnetism that go beyond mean-field Stoner models [7.8]. However, the first experiments left many open questions, particularly concerning the proper description of anisotropy energies, and whether the tunneling spectrum reflects the true electronic density of states or whether it is modified by non-equilibrium effects. To answer these questions, we have introduced a gate electrode to our devices and have also developed lower-noise tunnel barriers, thereby allowing new types of measurements and greatly improving the data quality. We present evidence that the quantum states in a ferromagnetic nanoparticle are not all described by the same anisotropy-energy function that governs the ground state [9], but the anisotropy varies from state to state. We show that non-equilibrium processes induced by tunneling affect the measured spectrum, thereby explaining a larger-than anticipated density of resonances. The gate electrode also allows a comparison with recent theories [7, 8] that minority-electron tunneling should dominate in a nanoparticle.

We will show data from both gated and non-gated devices. In either case, the samples contain a Co nanoparticle, separated from aluminum electrodes by aluminum oxide tunnel barriers, inside a tunnel junction with a nano-scale area small enough to allow individual particles to be contacted. (See device schematic, Fig. 1(a).) The devices without a gate electrode are fabricated
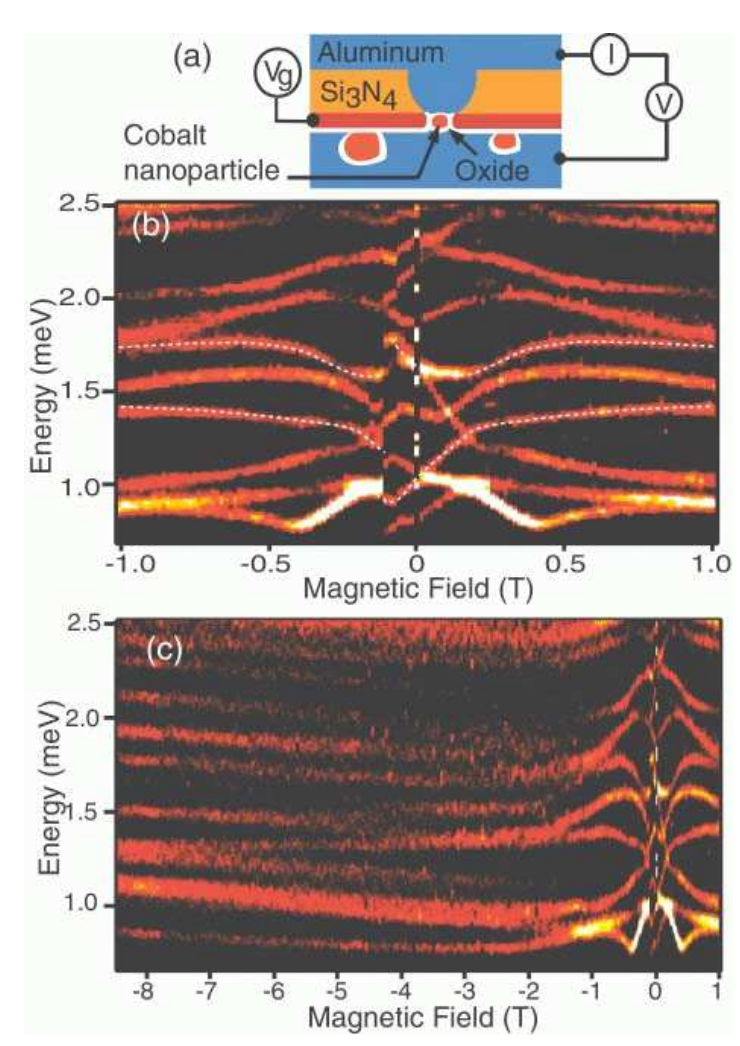

FIG. 1. (a) Cross-sectional device schematic. (b) and (c) Colorscale plots of $d I / d V$ for tunneling resonances in a cobalt nanoparticle. The field is varied from positive to negative values, and $d I / d V$ vs. $V$ is measured at each step. The maximum conductance is $3 \times 10^{-9} \Omega^{-1}$.

using a procedure described previously [6], with the new innovation that by using $1 \mathrm{~nm}$ of deposited aluminum oxide to form the tunnel barrier on the cobalt particle we produce junctions with reduced charge noise. The nanoparticles are made by depositing $0.5 \mathrm{~nm}$ of Co at room temperature, which makes particles in the range of $1-4 \mathrm{~nm}$ diameter [6]. The gated device is made by 
forming a hole about $10 \mathrm{~nm}$ in diameter in a suspended silicon-nitride membrane, depositing $18.5 \mathrm{~nm}$ of $\mathrm{Al}$ to make the gate electrode on the lower side of the device as shown in Fig. 1(a), and then isolating this gate by anodizing in an oxygen plasma to $3.5 \mathrm{~V}$ bias and depositing $8.5 \mathrm{~nm}$ of $\mathrm{SiO}_{\mathrm{x}}$. The rest of the fabrication proceeds in the same way as for the non-gated devices.

When cooled to dilution refrigerator temperatures, the tunneling conductance $d I / d V$ as a function of sourcedrain voltage $V$ consists of individual peaks, associated with transitions between discrete electronic states in the nanoparticle [6]. The $V$-spacings of resonances can be converted to energy, $\Delta E=e \Delta V C_{2} /\left(C_{1}+C_{2}\right)$, in this way correcting for capacitive division across the two junctions. The capacitance ratio can be determined by comparing peak positions at positive and negative $V$ [10,11. In Fig. 1(b,c), we plot the energies of tunneling resonances for a Co nanoparticle in a non-gated device as a function of magnetic field, $H$. As $H$ is swept from positive values toward zero, the levels first undergo significant continuous shifts. The discontinuity near $H=0$ is an artifact of the $\mathrm{Al}$ leads going superconducting and then being driven normal by a negative field. This causes the resonance energies to jump by $\Delta$, the superconducting gap [10], but the energies of the states within the nanoparticle evolve continuously. Near $\mu_{0} H_{\mathrm{sw}}=-0.120 \mathrm{~T}$, all the levels exhibit another large discontinuity, which can be identified with magnetic switching of the nanoparticle [6]. If $H$ is swept from negative to positive values, the field value for this transition is hysteretic, and the conductance spectrum is identical to that shown, but reflected about $H=0$. At larger values of $|H|$ (Fig. 1(c)), all the levels move to increasing energy, with slopes that correspond to effective $g$-factors ranging from 0.06 to 1.1. The fact that the $g$-factors are reduced below 2 indicates that the resonances are not purely spin-up or spin-down, but are mixed by spin-orbit scattering [12].

These data confirm in more detail several observations made previously [6]: the mean energy spacing is about $0.2 \mathrm{meV}$, smaller than estimated for independent electrons within a particle $1-4 \mathrm{~nm}$ in diameter; the energies are coupled to the orientation of the particle's magnetic moment; and resonances with a given high-field slope can jump either up or down at $H_{\mathrm{sw}}$. In addition, these data reveal several important new features. (1) More resonances are resolved than in the previous work, and it is clear that every level has a different $H$ dependence. (2) When the resonances approach close to each other as a function of $H$, they cross, with no evidence of avoidedcrossing behavior. (3) The levels can exhibit complicated non-monotonic variations as a function of $H$, for instance the levels marked with dots in Fig. 1(b).

The new observations are incompatible with the simplest model proposed previously for states in magnetic nanoparticles [6], which assumed that all the states in a particle could be described by a single anisotropy-

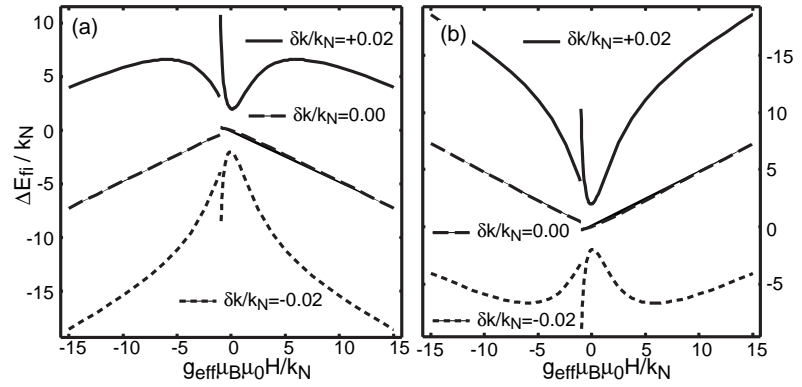

FIG. 2. Tunneling energy $\Delta E_{f i}$ calculated using the semiclassical model discussed in the text, with $H$ at $45^{\circ}$ from the easy axis, for various values of the anisotropy-energy difference $\delta k=k_{N+1}-k_{N}$. In (a) the total spin is increasing; in (b) it is decreasing. The curves are offset for clarity.

energy function. This assumption has the consequence that all transitions in which the spin component $\left\langle S_{z}\right\rangle$ increases should exhibit the same $H$ dependence, the $\left\langle S_{z}\right\rangle$ decreasing transitions should show a second behavior, and neither dependence should exhibit the complicated non-monotonicities below 1 Tesla evident in Fig. 1(b). (The results of the older model are shown in the $\delta k=0$ curves in Fig. 2.) The model in [6] assumed a uniaxial anisotropy, but we have confirmed that the same behaviors hold for more complicated forms which include terms of 2nd and 4th-order in the spin-components [9], as long as one assumes that the same anisotropy function applies to all electronic states.

The fact that all the tunneling resonances undergo different energy variations in the low- $H$ range where the magnetic moment is being reoriented indicates directly that all the electronic states of the particle cannot be described by the same anisotropy-energy function. We have explored whether such variations may also affect the form for the $H$-dependence of the energies as described in point (3) above, and we find that they provide a natural explanation for the complicated non-monotonic behavior as a function of $H$. We start by considering one single resonance associated with a transition between two states with $N$ and $N+1$ electrons. We extend the $N$ electron Hamiltonian stated in [6] in the simplest way to incorporate variations in anisotropy energy:

$$
\mathcal{H}=-g_{\mathrm{eff}} \mu_{B} \mu_{0} \vec{H} \cdot \vec{S}-k_{N}(\vec{S} \cdot \hat{n})^{2} / S_{0} .
$$

Here $\hbar \vec{S}$ is the total spin with ground-state magnitude $\hbar S_{0}$ for $N$ electrons, $\hat{n}$ is a unit vector in an easy-axis direction, and the (uniaxial) anisotropy energy prefactor $k_{N}$ is now allowed to vary between the $N$ - and $(N+1)$ electron states. For simplicity, we assume that the easy axis is the same for all states. We have solved for the ground state energies for $N$ and $N+1$ electrons semiclassically as a function of $H$ by finding the spin orientation that gives the local minimum in Eq. (1), assuming that $S_{0}$ does not vary with $H$ [7], and then we 
calculate the form of the tunneling transition energies as $E(N+1, H)-E(N, H)$. The results are shown in Fig. 2. Assuming $S_{0} \sim 1000$ (appropriate to a $4 \mathrm{~nm}$ Co particle), and an average value of $\left\langle k_{N}\right\rangle \approx 0.01 \mathrm{meV}$ in accord with the switching field [8,13], fluctuations in $k_{N}$ of order 1-3\% are sufficient to explain both the size and form of the non-monotonicities. Subsequent to this initial semiclassical analysis, similar conclusions were also reached in a more rigorous quantum-mechanical picture [8].

Although fluctuations in the properties of eigenstates are not often considered in the context of ferromagnets, they are not surprising. In non-magnetic particles, the $g$-factors for Zeeman splitting fluctuate [12], and the statistics of these fluctuations have been investigated in random-matrix treatments of the spin-orbit interaction [14,15. Anisotropy in magnetic particles also arises from spin-orbit interactions. An additional consequence of anisotropy-energy fluctuations should be that the value of $H_{\mathrm{sw}}$ will vary by $1-3 \%$ depending on the occupation of excited electronic states. This has yet to be investigated.

We now turn to data from a gated tunneling device, with the primary motivation being to resolve the question [6] of whether the larger-than-anticipated density of tunneling resonances observed at low energy (noted above) is due to inelastic emission of spin-waves during tunneling [16 or due to non-equilibrium effects [8, 17. The idea of the non-equilibrium mechanism is that the energy of tunneling transitions can be described as the energy differences of states in the nanoparticle with $N$ and $N \pm 1$ electrons: $\Delta E_{f i}^{ \pm} \equiv E_{f}^{N \pm 1}-E_{i}^{N}$. If under conditions of current flow, $E_{i}^{N}$ can assume an ensemble of different values, for instance due to low-energy spin excitations or electron-hole excitations within the particle, then the number of observed tunneling resonances can increase above the number that originate only from a single equilibrium ground state. We can test this with a gate voltage because the energy of a tunneling transition can be tuned from high values down close to zero where tunneling can be initiated by small $V$. For sufficiently small $V$, the tunneling electrons may have insufficient energy to excite non-equilibrium states. Therefore, a test of whether tunneling resonances are associated with non-equilibrium initial states is whether some transitions disappear when they are tuned to small $V$. This disappearance is exactly what is observed for the lowest-energy transitions at $H=0$ (Fig. 3(a)), when the electrodes are superconducting.

Related non-equilibrium effects have been observed in non-magnetic particles, but the consequences are much less dramatic. In $\mathrm{Al}$ particles, the energy shifts due to non-equilibrium are small, resulting only in a fine-structure about the energy of equilibrium transitions [17,18. We have not observed well-resolved transitions in non-magnetic particles to disappear completely as $V_{g}$ is varied, whereas at least the first 5
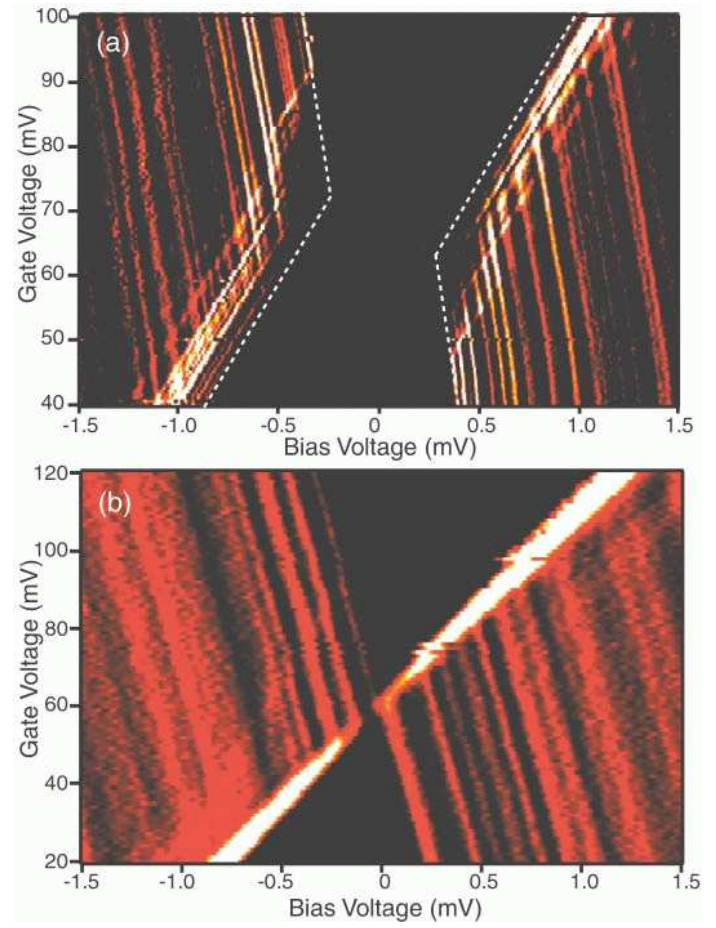

FIG. 3. Colorscale conductance plots of a gated Co nanoparticle as a function of $V_{g}$ and $V$. (a) Superconducting leads $(H=0)$. (b) Normal-state leads (0.07 Tesla). The dashed lines in (a) indicate the expected evolution of the threshold peaks if the resonances did not involve non-equilibrium processes. The voltage resolution is better at $H=0$ because of the superconducting singularity in the electrode density of states. The electron $T \approx 90 \mathrm{mK}$.

lowest-energy states in the Co particle lose conductance. Non-equilibrium effects therefore appear to be much stronger in Co, perhaps due to larger fluctuations in electron-electron interactions and/or a large multiplicity of low-energy collective spin excitations (in addition to the particle-hole excitations that were considered previously [17]). Since the relaxation rate of non-equilibrium excitations must be slower than the tunneling rate for the spectrum to be affected, the relaxation rate is of the order of or slower than $\sim 1 \mathrm{MHz}$.

The presence of level crossings (instead of avoided crossings), noted in Fig. 1 provides independent new evidence supporting the non-equilibrium scenario. In nonmagnetic particles, when spin-orbit scattering reduces the large- $H$ g-factors to less than 1.7, tunneling transitions originating from the same initial state exhibit clear level repulsion [12]. In contrast, under non-equilibrium conditions, tunneling resonances occurring at similar values of $V$ can result from different pairs of eigenstates $\left(E_{i}^{N}, E_{f}^{N \pm 1}\right)$, none of which are nearly degenerate, so an avoided crossing would not be expected.

Despite these two lines of evidence for the importance of non-equilibrium transitions, our observations are not 


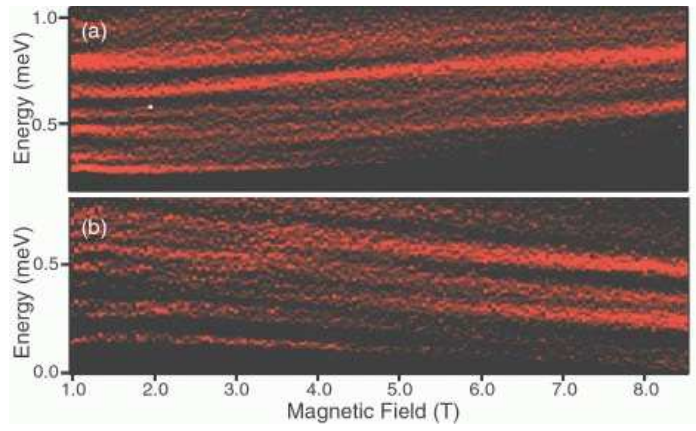

FIG. 4. High-field conductance spectra for the sample of Fig. 3 at (a) $V_{g}=20 \mathrm{mV}$ and (b) $V_{g}=125 \mathrm{mV}$.

in full agreement with the simplest phenomenological scenario that includes non-equilibrium effects [8]. Ref. [8] proposes that non-equilibrium spin-accumulation may occur by a sequence of transitions up a ladder of energy states having different total spin $S$, with the sequence terminating when the next step up the ladder requires more energy than that provided by the source-drain bias, $\mathrm{eV}$. Within this scenario, a fraction of both the low-energy and high-energy tunneling resonances should disappear as $V_{g}$ tunes the transitions to lower voltages. In Fig. 3(a), only the lowest-energy transitions experience a loss of tunneling amplitude. We suspect that a more complicated set of states than was used in [8] may need to be considered in characterizing non-equilibrium processes on our magnetic nanoparticle. For instance, single-electron quasiparticle excitations and also ladders of states with the same value of total spin $S$ but different values of $S_{z}$ may be involved.

Another unanticipated observation is that the disappearance of tunneling resonances as a function of $V_{g}$ occurs only when the electrodes are superconducting, and not when they are normal (Fig. 3(b)). Scans at 0.07, 1, 3,5 , and $8.5 \mathrm{~T}$ are all qualitatively similar. Perhaps, because of the singularity in the density of states for superconducting electrodes, the ensemble of non-equilibrium states may be populated differently by tunneling from normal and superconducting electrodes, with less nonequilibrium at low $V$ in the superconducting case. However, this process has not yet been modeled in detail.

One more simple benefit of having a gate electrode is that it allows a determination of whether the tunneling resonances correspond predominantly to the motion of majority or minority electrons. Consider the data shown in Figures 3 and 4 . At $V_{g}=20 \mathrm{mV}$ the low-energy tunneling thresholds correspond to the addition of an electron to the nanoparticle $(N \rightarrow N+1)$, and at large magnetic fields (Fig. 4(a)) the tunneling energies increase, meaning that the tunneling states have lower $\left\langle S_{z}\right\rangle$. It follows that the transitions correspond predominantly to minority-electron tunneling. Similarly, at $V_{g}=125 \mathrm{mV}$ (Fig. 4(b)), the tunneling thresholds are $N+1 \rightarrow N$ pro- cesses in which the spin increases, so a minority electron is tunneling off the nanoparticle. These results from one sample are in agreement with predictions [7] that the total spin should almost always decrease when electrons are added, due to both a large minority density of states and strong exchange interactions. Of course, because of spin-orbit coupling, the transitions are not purely of minority-electron character.

In summary, measurements of the $H$-dependence of the tunneling-resonance energies in cobalt nanoparticles exhibit level-to-level differences and non-monotonic variations that can both be explained by variations of $1-3 \%$ in magnetic anisotropy energy between different states. The observation of level crossings and the disappearance of resonances as a function of $V_{g}$ indicate that the majority of low-energy resonances are associated with tunneling transitions from non-equilibrium initial states. The strength of non-equilibrium effects appears to depend on whether the electrodes are normal or superconducting.

Acknowledgements: NSF DMR-0071631, DAAD19-011-0541, the Packard Foundation, the Cornell Nanofabrication Facility, and the DFG-Program "Semiconductor and Metallic Clusters".

[1] R. Meservey and P. M. Tedrow, Phys. Rep. 238, 173 (1994).

[2] K. Ono, H. Shimada, and Y. Ootuka, J. Phys. Soc. Jpn. 66, 1261 (1997); 672852 (1998).

[3] S. K. Upadhyay, A. Palanisami, R. N. Louie, and R. A Buhrman, Phys. Rev. Lett. 81, 3247 (1998).

[4] R. J. Soulen et al., Science 282, 85 (1999).

[5] J. M. De Teresa et al., Phys. Rev. Lett. 82, 4288 (1999).

[6] S. Guéron, M. M. Deshmukh, E. B. Myers, and D. C. Ralph, Phys. Rev. Lett. 83, 4148 (1999).

[7] C. M. Canali, and A. H. MacDonald, Phys. Rev. Lett. 85, 5623 (2000).

[8] S. Kleff, J. von Delft, M. M. Deshmukh, and D. C. Ralph, cond-mat/0103626.

[9] A. Thiaville, Phys. Rev. B 61, 12221 (2000).

[10] D. C. Ralph, C. T. Black, and M. Tinkham, Phys. Rev. Lett. 74, 3241 (1995).

[11] For the sample of Fig. 1, $C_{1} / C_{2}=0.42$, and for Figs. 3,4, $C_{1} / C_{2}=0.25$.

[12] D. G. Salinas et al., Phys. Rev. B 60, 6137 (1999).

[13] The estimate $\left\langle k_{N}\right\rangle \sim 0.1 \mathrm{meV}$ in [6] based on the size of energy-level jumps at $H_{\mathrm{sw}}$ is inaccurate because it neglects effects of $k_{N}$-fluctuations [8].

[14] K. A. Matveev, L. I. Glazman, and A. I. Larkin, Phys. Rev. Lett. 85, 2789 (2000).

[15] P. W. Brouwer, X. Waintal, and B. I. Halperin, Phys. Rev. Lett. 85, 369 (2000).

[16] S. Zhang, P. M. Levy, A. C. Marley, and S. S. P. Parkin Phys. Rev. Lett. 79, 3744 (1997).

[17] O. Agam et al., Phys. Rev. Lett. 78, 1956 (1997).

[18] M. M. Deshmukh et al., cond-mat/0106024. 